TIFR/TH/05-42

NSF-KITP-05-83

hep-th/0510104

\title{
Closed string tachyons, flips and conifolds
}

\author{
K. Narayan \\ Department of Theoretical Physics, \\ Tata Institute of Fundamental Research, \\ Homi Bhabha Road, Colaba, Mumbai - 400005, India. \\ Email: narayan@theory.tifr.res.in
}

\begin{abstract}
Following the analysis of tachyons and orbifold flips described in hep-th/0412337, we study nonsupersymmetric analogs of the supersymmetric conifold singularity and show using their toric geometry description that they are nonsupersymmetric orbifolds of the latter. Using linear sigma models, we see that these are unstable to localized closed string tachyon condensation and exhibit flip transitions between their two small resolutions (involving 2-cycles), in the process mediating mild dynamical topology change. Our analysis shows that the structure of these nonsupersymmetric conifolds as quotients of the supersymmetric conifold obstructs the 3-cycle deformation of such singularities, suggesting that these nonsupersymmetric conifolds decay by evolving towards their stable small resolutions.
\end{abstract}




\section{Contents}

\begin{tabular}{lll}
\hline & Introduction & 1
\end{tabular}

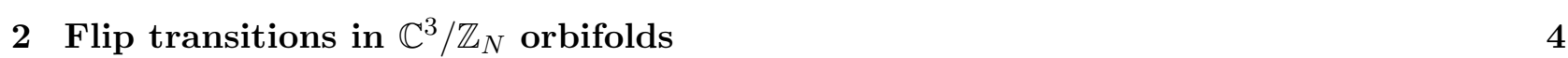

3 Tachyons and flip conifolds $\quad 8$

3.1 An example: $(12-1-3) \ldots \ldots \ldots \ldots$

3.2 The geometrv of the $\left(n_{1} n_{2}-n_{2}-n_{1}\right)$ flip conifold region . . . . . . . . . 10

3.2 .1 On deformations of the singularity . . . . . . . . . . . . . . 13

3.3 Small resolutions and their dvnamics . . . . . . . . . . . . . . . . . 16

$\begin{array}{lll}4 & \text { Discussion } & 22\end{array}$

$\begin{array}{ll}\text { A More on deformations } & 25\end{array}$

\section{Introduction}

The study of closed string tachyon condensation in unstable geometries in string theory, beginning with [1], followed by [2], [3], and others, has been quite rich (see e.g. the reviews [4] [5]), from the point of view of understanding the vacuum structure of string theory, the role of time, as well as phenomena pertaining to quantum geometry. While direct (second order) time evolution is often hard to study, considerable qualitative insight is gained by studying (first order) renormalization group flows in appropriate auxiliary 2-dimensional (worldsheet) theories describing the unstable system: these are often tractable when worldsheet supersymmetry is unbroken, spacetime supersymmetry being broken, and are then closely intertwined with the algebro-geometric structure of the singularities and their resolutions. Closed string tachyons localized at singularities, somewhat analogous to open string tachyons localized on brane-antibrane defects in a fixed ambient spacetime, are simpler than delocalized instabilities since initially they affect only the vicinity of the singularity: in fact, their condensation often turns out to resolve the singularity.

Closed string tachyons localized at $\mathbb{C}^{3} / \mathbb{Z}_{N}$ nonsupersymmetric orbifold singularities were studied in [8] (see also [10]), with a more detailed gauged linear sigma model (GLSM) analysis of dynamical topology change via flip transitions given in [9]. Codimension three singularities are somewhat complicated due to the existence of terminal singularities and the absence of canonical resolutions. The analysis of possible endpoints in [8] involved studying the decay channel corresponding to the condensation of the most relevant tachyon and sequentially iterating this procedure for each of the residual geometries, which are themselves typically 
unstable. The generic endpoints of decay for Type II string propagation on such singularities are smooth, i.e. spaces with at worst supersymmetric singularities that can be resolved by moduli (marginal operators); however, Type 0 theories do in fact generically exhibit a terminal singularity $\mathbb{C}^{3} / \mathbb{Z}_{2}(1,1,1)$ in their spectrum of decay endpoints. Generically there are multiple distinct tachyons. This is particularly important for codimension three singularities due to the absence of canonical resolutions: the various possible distinct resolutions are related by flips and flops, mediated by relevant and marginal operators, respectively, in the worldsheet theory. The competition between tachyons of distinct R-charges (i.e. masses in spacetime) gives rise to flips [9], described in part in [8], relating the distinct partial resolutions of the original singularity, thought of as distinct attractor basins for the worldsheet RG flow. A flip transition can be thought of as a blowdown of a cycle accompanied by a blowup of a topologically distinct cycle: this involves a mild change in the topology of the ambient (embedding) compact space containing such local singularities, with changes in the intersection numbers of the various cycles of the geometry, the two cycles being topologically distinct (although the analogs of the Hodge numbers, i.e. the numbers of $p$-cycles, themselves do not change, as for supersymmetric flops in Calabi-Yau spaces [11] [12]). Orbifold flips [9] can always be consistently embedded in a 2-tachyon sub-GLSM of the full, say, $n$-tachyon GLSM, reflecting the fact that in the corresponding toric fan, they occur in subcones containing a reversal of the sequence of subdivisions pertaining to only one wall in the fan: we will refer to this subcone containing the nontrivial flip dynamics as a flip region. Physically a flip occurs when a more dominant tachyon condenses during the condensation of some tachyon, and the effective dynamics of the relative condensation process can be described by a GLSM with gauge group $U(1)$, as we will review later. In particular the dynamics of the GLSM RG flow always drives a flip transition in the direction of the partial resolution leading to a less singular residual geometry, which can be thought of as a more stable endpoint: this enables a classification of the phases of GLSMs corresponding to these unstable orbifolds into "stable" and "unstable" basins of attraction, noting the directionality of the RG trajectories involving potential flip transitions, which always flow towards the more stable phases.

We mention here in passing that D-branes and Coulomb branches in $\mathbb{C}^{2} / \mathbb{Z}_{N}$ singularities have been studied most recently in [15] following [6, 7], while [13, 16] study the emergence of Coulomb branches in codimension three. [14] describes topology-changing transitions mediated by tachyons in string compactifications on Riemann surfaces. See also e.g. [17, 18, 19, 20, 21, 22, 23, 24, 25], for other interesting recent work pertaining to closed string tachyons.

As mentioned above (and will be reviewed in more detail in Sec. 2), an orbifold flip occurs within a flip region representing a 2-tachyon sub-GLSM and the condensation of the tachyons "relative" to each other can be described by an effective GLSM with gauge group $U(1)$ : in 
this case, the effective dynamics of the flip region is simply a part of the full decay structure of the orbifold, the corresponding subcone being embedded in the full toric fan of the orbifold. In this work, we study these flip regions as singularities in themselves, devoid of the orbifold embedding, and analyze their dynamics. From their toric data, we show that they are unstable conifold-like singularities, obtained as nonsupersymmetric orbifolds of the supersymmetric conifold. They are labelled by a charge matrix

$$
Q=\left(\begin{array}{llll}
n_{1} & n_{2} & -n_{3} & -n_{4}
\end{array}\right), \quad \Delta n \equiv \sum Q_{i} \neq 0
$$

for integers $n_{i}>0$, which characterizes their toric data and therefore their geometry (in this notation, the supersymmetric conifold corresponds to $\left.Q=\left(\begin{array}{llll}1 & 1 & -1 & -1\end{array}\right)\right)$. As orbifolds of the supersymmetric conifold, they can be described by a hypersurface equation $z_{1} z_{4}-z_{2} z_{3}=0$, with the new feature that the $z_{i}$ are coordinates not in $\mathbb{C}^{4}$ but in $\mathbb{C}^{4} / \Gamma$ where $\Gamma$ is a discrete group depending on the $n_{i}$. Throughout our discussion, we view such a geometry as the vicinity of a local 3-complex dimensional singularity embedded in some compact space (possibly some appropriate nonsupersymmetric orbifold of a Calabi-Yau that develops a supersymmetric conifold singularity) and focus on the local dynamics of the singularity, the full spacetime in this effective noncompact limit being of the form $\mathbb{R}^{3,1} \times \mathcal{C}^{(f l i p)}$. With a view to understanding the dynamics in our nonsupersymmetric case here, somewhat analogous to [27] [28] (see also the review [29]) for the supersymmetric conifold, we study the geometry in the vicinity of the singularity in Sec. 3, in part drawing analogies with the corresponding analysis [26] of the supersymmetric conifold. We recall that the supersymmetric conifold admits two topologically distinct small resolutions replacing the singularity by 2-cycles (Kähler deformations related by a flop) and a complex structure deformation replacing the singularity by a 3-cycle. In the case at hand, our analysis shows that these 3-cycle deformations are obstructed for such flip conifold singularities due to their structure as quotients of the supersymmetric conifold ${ }^{1}$, suggesting that the decay structure of these singularities is always via their small resolutions, involving 2 -cycles. Unlike a flop, the fact that $\sum Q_{i} \neq 0$ gives rise to an asymmetry in the resolutions, so that one of the resolutions has a spontaneous tendency to evolve towards the other, more stable, one: thus there is an inherent directionality here, leading to dynamical topology change with a blowdown of a 2-cycle followed by a spontaneous blowup of the other (topologically distinct) 2-cycle. We study the dynamics of the small resolutions of these singularities using linear sigma models, gaining insight from the RG dynamics of the Fayet-Iliopoulos parameter. As in orbifold decay where divisors blow up and expand in time, potentially containing unstable residual singularities on their loci, the 2-cycles in the small resolution decay channels of these

\footnotetext{
${ }^{1}$ Note that this does not however preclude abstract deformations.
} 
flip conifolds potentially contain unstable residual orbifold singularities which then themselves decay. Using the Type II GSO projection for these residual orbifold singularities, we find a nontrivial constraint $\Delta n=$ even for the $\mathbb{R}^{3,1} \times \mathcal{C}^{(\text {flip) }}$ spacetime background to admit a Type II GSO projection with no bulk tachyons: this is consistent with the endpoint orbifold singularities also admitting Type II GSO projections. Finally in Sec. 4 we conclude with a brief discussion on the evolution of these geometries.

\section{$2 \quad$ Flip transitions in $\mathbb{C}^{3} / \mathbb{Z}_{N}$ orbifolds}

The closed string tachyon dynamics of nonsupersymmetric $\mathbb{C}^{3} / \mathbb{Z}_{N}$ orbifolds has been studied in some detail in [8] $[9]^{2}$. The absence of canonical (minimal) resolutions gives rise to flips.

To illustrate these phenomena, we review a concrete example $\mathbb{C}^{3} / \mathbb{Z}_{13}(1,2,5)$ and the flip dynamics here, studied in detail in [9]. This can be described by a GLSM with gauge group $U(1) \times U(1)$ and two of five chiral superfields representing tachyons $T_{1}$ and $T_{8}$ with masses $m_{1}^{2}=\frac{2}{\alpha^{\prime}}\left(\frac{8}{13}-1\right)$ and $m_{8}^{2}=\frac{2}{\alpha^{\prime}}\left(\frac{11}{13}-1\right)$ respectively, their R-charges given by $R_{1} \equiv\left(\frac{1}{13}, \frac{2}{13}, \frac{5}{13}\right)=\frac{8}{13}$ and $R_{8} \equiv\left(\frac{8}{13}, \frac{3}{13}, \frac{1}{13}\right)=\frac{12}{13}$. The action of the GLSM is (we use the conventions of [12, 31])

$$
S=\int d^{2} z\left[d^{4} \theta\left(\bar{\Psi}_{i} e^{2 Q_{i}^{a} V_{a}} \Psi_{i}-\frac{1}{4 e_{a}^{2}} \bar{\Sigma}_{a} \Sigma_{a}\right)+\operatorname{Re}\left(i t_{a} \int d^{2} \tilde{\theta} \Sigma_{a}\right)\right]
$$

summation over $a=1,2$, being implied. The $t_{a}=i r_{a}+\frac{\theta_{a}}{2 \pi}$ are Fayet-Iliopoulos parameters and $\theta$-angles for both the gauge fields, $e_{a}$ being the gauge couplings. The twisted chiral superfields $\Sigma_{a}$ (whose bosonic components include complex scalars $\sigma_{a}$ ) represent field-strengths for the gauge fields. The action of the $U(1)^{2}$ on the chiral superfields $\Psi \equiv \phi_{1}, \phi_{2}, \phi_{3}, T_{1}, T_{8}$, comprising the three coordinate superfields and the two tachyons, is given by $\Psi_{i} \rightarrow e^{i Q_{i}^{a} \lambda} \Psi_{i}$, where the charge matrix is

$$
Q_{i}^{a}=\left(\begin{array}{ccccc}
1 & 2 & 5 & -13 & 0 \\
8 & 3 & 1 & 0 & -13
\end{array}\right), \quad i=1, \ldots, 5, \quad a=1,2 .
$$

Such a charge matrix only specifies the $U(1) \times U(1)$ action up to a finite group, due to the possibility of a $\mathbb{Q}$-linear combination of the rows of the matrix also having integral charges. There are two independent FI parameters (for the two $U(1)$ 's) whose variations control the vacuum structure of the theory. The space of classical ground states of this theory can be found from the bosonic potential

$$
U=\sum_{a} \frac{\left(D_{a}\right)^{2}}{2 e_{a}^{2}}+2 \sum_{a, b=1}^{2} \bar{\sigma}_{a} \sigma_{b} \sum_{i} Q_{i}^{a} Q_{i}^{b}\left|\Psi_{i}\right|^{2}
$$

\footnotetext{
${ }^{2}$ See also [10] [13].
} 


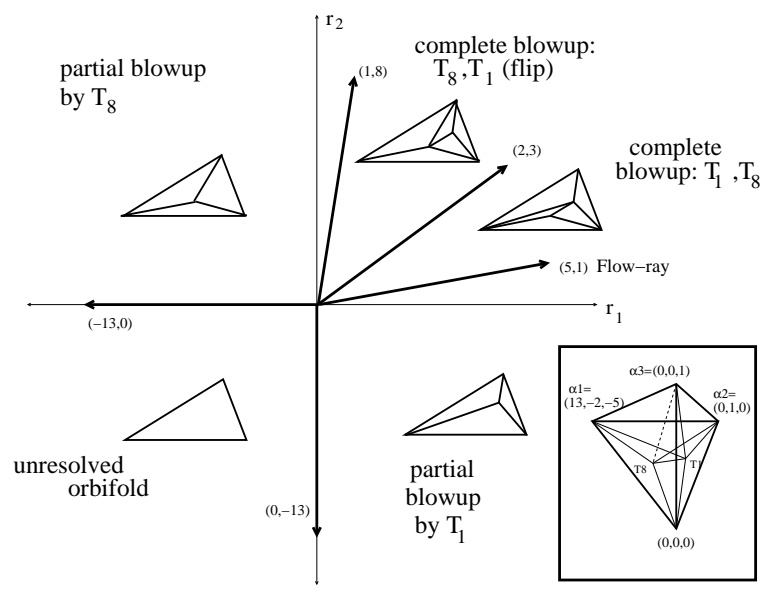

Figure 1: The phase structure of $\mathbb{C}^{3} / \mathbb{Z}_{13}(1,2,5)$ for two tachyons. The inset shows the toric fan and its subdivisions.

Then $U=0$ requires $D_{a}=0$ : solving these for $r_{a} \neq 0$ gives expectation values for the $\Psi_{i}$, which Higgs the gauge group down to some discrete subgroup and lead to mass terms for the $\sigma_{a}$ whose expectation values thus vanish. The classical vacua of the theory are then given in terms of solutions to the D-term equations

$$
\frac{-D_{a}}{e^{2}}=\sum_{i} Q_{i}^{a}\left|\Psi_{i}\right|^{2}-r_{a}=0, \quad a=1,2
$$

These give collections of coordinate charts that characterize in general distinct toric varieties. In other words, this 2-parameter system admits several "phases" (convex hulls in $r$-space) depending on the values of $r_{1}, r_{2}$, shown in Figure 1 . In this case, there are five phases, corresponding to the unresolved orbifold, the partial resolutions by condensation of $T_{1}$ or $T_{8}$ alone, and the complete resolutions by condensation of both $T_{1}$ and $T_{8}$. Note that the order in which the tachyons condense changes the resulting endpoint: condensation of $T_{8}$ followed by $T_{1}$ gives rise to an endpoint geometry that is related by a flip transition (blowdown+blowup) to that obtained by condensation of the sequence $T_{1}, T_{8}$. Each phase is an endpoint since if left unperturbed, the corresponding resolution of the singularity can continue indefinitely (within this noncompact approximation): in this sense, each phase (more accurately, an appropriate limit inside the phase) is a fixed point of the GLSM RG flow. However some of these phases are unstable while others are stable, in the sense that small fluctuations (condensation of other tachyons) will cause the system to run away from the unstable phases towards the stable ones. This can be gleaned from the 1-loop renormalization of the FI parameters

$$
r_{a}=\left(\frac{\sum_{i} Q_{i}^{a}}{2 \pi}\right) \log \frac{\mu}{\Lambda}
$$


where $\mu$ is the RG scale and $\Lambda$ is an ultraviolet cutoff scale where the $r_{a}$ are defined to vanish. We restrict attention to the large $r_{a}$ regions, thus ignoring worldsheet instanton corrections, which is sufficient for our purposes of understanding the phase structure. A generic linear combination of the gauge fields couples to a linear combination of the FI parameters and, in this example, has the 1-loop running

$$
c_{1} r_{1}+c_{2} r_{2}=-\left(\frac{5 c_{1}+c_{2}}{2 \pi}\right) \log \frac{\mu}{\Lambda}
$$

This parameter is marginal if $5 c_{1}+c_{2}=0$ : this describes a line perpendicular to the ray $(5,1)$ in $r$-space (i.e. the secondary fan). Thus we can choose the basis for the $U(1) \times U(1)$ so that one linear combination couples to a (relevant) FI parameter that has nontrivial renormalization along the flow, while the other FI parameter is marginal along the flow. From (17), we see that this single relevant direction (perpendicular to the marginal one), lies along the ray $(5,1)$ : this is the flow-ray. These equations indicate that at low energies $\mu \ll \Lambda$, the 1-loop RG flows drive the system towards the large $r$ regions in $r$-space, i.e., $r_{1}, r_{2} \gg 0$, that are adjacent to the flow-ray $(5,1)$ : these phases, i.e. the convex hulls $\{(5,1),(0,-13)\}$ and $\{(2,3),(5,1)\}$, are thus the stable attractors for the GLSM RG flow. For generic combinatorics, the flow-ray lies in the interior of some convex hull in the secondary fan, in which case there is precisely one stable phase. However if the flow-ray is the boundary of two adjacent convex hulls, as it is here, there will be multiple stable phases related by marginal deformations or moduli in the infrared. From Figure 1, we see that the two stable phases arising as the endpoints of flowlines correspond to the partial resolution by the tachyon $T_{1}$ alone and the complete resolution by the tachyon $T_{1}$ followed by $T_{8}$. This dovetails nicely with the toric analysis in [8]: after condensation of $T_{1}$ (the most relevant tachyon here), the subsequent tachyon $T_{8}$ becomes massless so that the blowup it corresponds to is now a modulus or flat direction, rather than a residual instability. This marginality of the residual (erstwhile tachyon) $T_{8}$ reflects the marginal $U(1)$ direction perpendicular to the flow-ray $(5,1)$ in the GLSM. Thus the GLSM RG flow drives the system along the flow-ray, maintaining a flat direction perpendicular to it.

In the process of ending up in one of these stable phases, flowlines may cross one or more of the the (semi-infinite) phase boundaries emanating from $(0,0)$ and passing through $(-13,0),(1,8),(2,3)$ and $(0,-13)$. In particular in crossing $(2,3)$, a flip occurs, involving a reversal of the sequence of partial resolutions of the orbifold by the two tachyons, from $T_{8}$ followed by $T_{1}$, to $T_{1}$ and then $T_{8}$. The physics of this phase boundary occurs in the region of the 2D moduli space where the nontrivial dynamics does not involve the field $\phi_{2}$, enabling us to study the effective dynamics of the flip transition captured by this subsystem, the flip region here being defined by the subcone $T_{1}, T_{8}, \phi_{1}, \phi_{3}$ (see Figure 1). This is described by an 
effective $U(1)$ GLSM with the effective D-term

$$
\frac{-D^{e f f}}{13 e^{2}}=-\frac{1}{13}\left(3 D_{1}-2 D_{2}\right)=\left|\phi_{3}\right|^{2}+2\left|T_{8}\right|^{2}-\left|\phi_{1}\right|^{2}-3\left|T_{1}\right|^{2}-r^{e f f}=0,
$$

the effective charge matrix being $Q^{\text {eff }}=\left(\begin{array}{llll}1 & 2 & -1 & -3\end{array}\right)$. This effective Fayet-Iliopoulos parameter $r^{e f f}=\frac{3 r_{1}-2 r_{2}}{13}$ has a 1-loop renormalization given by

$$
r^{e f f}=\left(\sum_{i} Q_{i}^{e f f}\right) \log \frac{\mu}{\Lambda}=(-1) \log \frac{\mu}{\Lambda}
$$

showing the inherent directionality of the flip in spacetime. Thus $r^{\text {eff }}$ flows under the GLSM renormalization group from the $r^{e f f} \ll 0$ phase (partial resolution by a 2-cycle with coordinate charts $\left.\left(\phi_{3}, T_{8}, \phi_{1}\right),\left(\phi_{3}, T_{8}, T_{1}\right)\right)$ to the $r^{e f f} \gg 0$ phase (partial resolution by a different 2-cycle with charts $\left.\left(\phi_{3}, \phi_{1}, T_{1}\right),\left(T_{8}, \phi_{1}, T_{1}\right)\right)$ which has distinct topology.

This is a fairly generic story for flip dynamics in unstable orbifolds, described in generality in 9]. We recall [8, 9] that the cumulative degree of the residual singularities after condensation of a tachyon $T_{j}$ with R-charge $R_{j}$, i.e. subdivision by the corresponding lattice point in the toric cone, is given by the total $\boldsymbol{N}$ lattice volume $V\left(T_{j}\right)=N R_{j}$ of the residual subcones: thus a more relevant tachyon gives rise to a smaller $\boldsymbol{N}$ lattice volume, i.e. a less singular residual endpoint. The difference in the subcone volumes in the two partial resolutions (see Figure 1)

$$
\Delta V=V\left(0 ; \alpha_{1}, T_{1}, \alpha_{3}\right)+V\left(0 ; \alpha_{3}, T_{1}, T_{8}\right)-V\left(0 ; \alpha_{1}, T_{1}, T_{8}\right)-V\left(0 ; \alpha_{1}, T_{8}, \alpha_{3}\right)
$$

can be thought of as a quantitative measure of the difference in the degree of singularity of the two resolutions. Furthermore the coefficient of the logarithm in $r^{\text {eff }}$ turns out to be

$$
r^{e f f}=(\Delta V) \cdot \log \frac{\mu}{\Lambda} .
$$

Thus the RG flow for this effective FI parameter proceeds precisely in the direction of decreasing $\boldsymbol{N}$ lattice volume, i.e., in the direction along which $\Delta V<0$. In other words, the GLSM RG flow dynamics drives the flip transition in the direction of the less singular residual geometry, which in this example is the stable phase corresponding to the blowup sequence $T_{1}, T_{8}$.

The 1-loop renormalization of the FI parameters can be expressed [12, 30, 31] in terms of a perturbatively quantum-corrected twisted chiral superpotential for the $\Sigma_{a}$

$$
\tilde{W}(\Sigma)=\frac{1}{2 \sqrt{2}} \sum_{a=1}^{n} \Sigma_{a}\left(i \hat{\tau}_{a}-\frac{1}{2 \pi} \sum_{i=1}^{d+n} Q_{i}^{a} \log \left(\sqrt{2} \sum_{b=1}^{n} Q_{i}^{b} \Sigma_{b} / \Lambda\right)\right)
$$

for a general $n$-tachyon system, obtained by considering the large- $\sigma$ region in field space and integrating out those scalars $\Psi_{i}$ that are massive here (and their expectation values vanish 
energetically). This leads to the modified potential

$$
U(\sigma)=\frac{e^{2}}{2} \sum_{a=1}^{n}\left|i \hat{\tau}_{a}-\frac{\sum_{i=1}^{d+n} Q_{i}^{a}}{2 \pi}\left(\log \left(\sqrt{2} \sum_{b=1}^{n} Q_{i}^{b} \sigma_{b} / \Lambda\right)+1\right)\right|^{2} .
$$

The singularities predicted classically at the locations of the phase boundaries arise from the existence of low-energy states at large $\sigma$. Now from above, we see that along the single relevant direction where $\sum_{i} Q_{i}^{1} \neq 0$, the potential energy has a $\left|\log \left(\sigma_{1}\right)\right|^{2}$ growth so that the field space accessible to very low-lying states is effectively compact and there is no singularity along the single relevant direction given by the flow-ray [9]: in other words, the RG flow is smooth along the tachyonic directions for all values of $\tau_{1}$, and the phase boundaries are simply labels for the boundaries of the adjacent phases.

\section{Tachyons and flip conifolds}

In the previous section, the flip regions in question were embedded within nonsupersymmetric $\mathbb{C}^{3} / \mathbb{Z}_{N}$ orbifold singularities. In what follows, we will study the singularity structure of flip regions treating them as geometric objects in their own right, described by their toric data. In particular, from the toric fan of a given flip region, we can glean the algebraic structure of the corresponding singularity. We will see that these flip regions are to be thought of as nonsupersymmetric analogs of the supersymmetric conifold singularity.

Let us first recall some key features of the supersymmetric conifold (the description below of the toric data, the corresponding $U(1)$ action and algebraic singularity structure for the supersymmetric conifold can be found in e.g. [32]): this can be represented by a $\mathbb{C}^{*}$ action given by the charge matrix

$$
Q=\left(\begin{array}{llll}
1 & 1 & -1 & -1
\end{array}\right)
$$

with action $\Psi_{i} \rightarrow \lambda^{Q_{i}} \Psi_{i}, \lambda \in \mathbb{C}^{*}$, on the coordinates $\Psi_{i} \equiv a, b, c, d$, describing the singularity. The supersymmetric conifold is specified by toric data (see Figure 2) given by four (minimal) lattice points $e_{i}$ such that $e_{1}+e_{2}-e_{3}-e_{4}=0$, the coefficients being fixed by the charges $Q_{i}$. A basis of monomials invariant under the $\mathbb{C}^{*}$ action is $z_{1}=a c, z_{2}=a d, z_{3}=b c, z_{4}=b d$, satisfying

$$
z_{1} z_{4}-z_{2} z_{3}=0
$$

which describes the supersymmetric conifold as a hypersurface ${ }^{3}$ embedded in $\mathbb{C}^{4}$. Now recall that the supersymmetric conifold singularity above can be smoothed out in two distinct ways: $(i)$ via either of the two small resolutions of the singularity obtained by blowing up two-spheres

\footnotetext{
${ }^{3}$ This can be recast as $\sum_{i=1}^{4} w_{i}^{2}=0$, by appropriate redefinitions of the coordinates $z_{i}$.
} 
$\left(\mathbb{P}^{1} \mathrm{~s}\right)$ at the singularity - these are Kähler deformations, related by a flop transition; ( $\left.i i\right)$ via a complex structure deformation of the hypersurface equation as $z_{1} z_{4}-z_{2} z_{3}=\epsilon$, obtained by blowing up a three-sphere.

In what follows, we study along the above lines the geometry of flip regions.

\subsection{An example: $\left(\begin{array}{llll}1 & 2 & -1 & -3\end{array}\right)$}

For instance, let us revisit the flip region in $\mathbb{C}^{3} / \mathbb{Z}_{13}(1,2,5)$ : a basis for the $\boldsymbol{N}$ lattice containing the tetrahedral flip region consists of the vertices $e_{3} \equiv \phi_{3}=(0,0,1), e_{1} \equiv T_{1}=(1,0,0), e_{2} \equiv$ $T_{8}=(0,1,0)$, relabelling the tachyonic lattice point $T_{8}=(8,-1,-3)$ for convenience (see Figure 1). Then the coordinate vertex $\phi_{1}=(13,-2,-5) \equiv e_{4}$ in terms of the $e_{1}, e_{2}, e_{3}$ basis satisfies the relation $e_{4}+3 e_{1}-2 e_{2}-e_{3}=0$, which is suggestively reminiscent of the equation describing the supersymmetric conifold as a hypersurface in $\mathbb{C}^{4}$. Let us now strip off the orbifold embedding and study this flip region as a geometric object in itself (see Figure 21): with this in mind, let us realize the algebraic structure of the flip from its toric data. The $\mathbb{C}^{*}$ action here on $a, b, c, d$, is specified by the charge matrix

$$
Q=\left(\begin{array}{llll}
1 & 2 & -1 & -3
\end{array}\right)
$$

Then the redefined coordinates $a, b^{\frac{1}{2}}, c, d^{\frac{1}{3}}$ have a new $\mathbb{C}^{*}$ action specified by the matrix $Q=\left(\begin{array}{llll}1 & 1 & -1 & -1\end{array}\right)$. Defining the monomials $z_{1}=a c, z_{2}=a d^{\frac{1}{3}}, z_{3}=b^{\frac{1}{2}} c, z_{4}=b^{\frac{1}{2}} d^{\frac{1}{3}}$, invariant under the new $\mathbb{C}^{*}$ action, it is straightforward to see that the $z_{i}$ satisfy the relation $z_{1} z_{4}-z_{2} z_{3}=0$, i.e. the conifold equation (15).

However note that now there is a residual discrete $\mathbb{Z}_{6}$ acting nontrivially on the $z_{i}$, stemming from the well-defined geometric rotation by $e^{2 \pi i}$ on the coordinates $a, b, c, d$. The action of the $\mathbb{Z}_{6}$ is

$$
\left(\begin{array}{cccc}
z_{1} & z_{2} & z_{3} & z_{4}
\end{array}\right) \rightarrow\left(\begin{array}{cccc}
z_{1} & \omega^{1 / 3} z_{2} & \omega^{1 / 2} z_{3} & \omega^{5 / 6} z_{4}
\end{array}\right), \quad \omega=e^{2 \pi i},
$$

which can be rewritten in terms of $\omega^{\prime}=e^{2 \pi i / 6}$. In other words, the $z_{i}$ as coordinates are well-defined only up to these discrete identifications: we will elaborate on this description in the next subsection. Thus the geometry of this flip conifold is somewhat different from the supersymmetric conifold, which has no such discrete symmetry. In this case, the flip conifold is a hypersurface in $\mathbb{C}^{4} / \mathbb{Z}_{6}$ with the $\mathbb{Z}_{6}$ action (17). This is a generic feature of such tachyonic conifolds exhibiting nonsupersymmetric flip regions, as we will study in greater detail below.

From (17), we can see that each of the two terms in the hypersurface equation (15) for the flip conifold above has a nontrivial action of the $\mathbb{Z}_{6}$, given by the phase $e^{2 \pi i(5 / 6)}$. Thus there is an obstruction to the existence of a 3-cycle $\left(S^{3}\right)$ deformation of the hypersurface 
equation of the form $z_{1} z_{4}-z_{2} z_{3}=\epsilon \neq 0$, since the deformation parameter $\epsilon$ can be chosen a real number (by coordinate changes) trivial under $\mathbb{Z}_{6}$ and does not respect the $\mathbb{Z}_{6}$ symmetry of the original singularity. Roughly speaking, such a deformed geometry lies "outside" the symmetry-preserving phases of the geometry that are connected to the singular point itself.

In what follows, we will study the geometry of arbitrary flip conifolds using their toric data along the lines above.

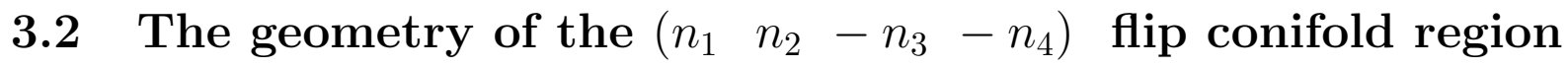

Consider a charge matrix

$$
Q=\left(\begin{array}{llll}
n_{1} & n_{2} & -n_{3} & -n_{4}
\end{array}\right)
$$

and a $\mathbb{C}^{*}$ action on the complex coordinates $\Psi_{i} \equiv a, b, c, d$, with this charge matrix as $\Psi_{i} \rightarrow$ $\lambda^{Q_{i}} \Psi_{i}, \lambda \in \mathbb{C}^{*}$. Since we have defined the minus signs to be in specific places in $Q$, the $\left\{n_{i}\right\}$ must be treated as an ordered set. For an unstable (tachyonic) geometry, we have $\sum_{i} Q_{i} \neq 0$. The flip region corresponding to this $Q$ can be described, as in Figure 2 , by a toric cone defined by the lattice vectors $e_{i}$ satisfying the relation

$$
\sum Q_{i} e_{i}=n_{1} e_{1}+n_{2} e_{2}-n_{3} e_{3}-n_{4} e_{4}=0
$$

in a 3-dimensional $\boldsymbol{N}$ lattice. Then the redefined coordinates $a^{\frac{1}{n_{1}}}, b^{\frac{1}{n_{2}}}, c^{\frac{1}{n_{3}}}, d^{\frac{1}{n_{4}}}$ have a new $U(1)$ action given by $\tilde{Q}=\left(\begin{array}{llll}1 & 1 & -1 & -1\end{array}\right)$. Defining the invariant monomials

$$
z_{1}=a^{\frac{1}{n_{1}}} c^{\frac{1}{n_{3}}}, \quad z_{2}=a^{\frac{1}{n_{1}}} d^{\frac{1}{n_{4}}}, \quad z_{3}=b^{\frac{1}{n_{2}}} c^{\frac{1}{n_{3}}}, \quad z_{4}=b^{\frac{1}{n_{2}}} d^{\frac{1}{n_{4}}}
$$

we see that the $z_{i}$ satisfy

$$
z_{1} z_{4}-z_{2} z_{3}=0
$$

i.e. the conifold equation (15). Each of the two terms on the left hand side of this conifold equation now has acting on it a discrete phase,

$$
e^{2 \pi i\left(\frac{1}{n_{1}}+\frac{1}{n_{2}}+\frac{1}{n_{3}}+\frac{1}{n_{4}}\right)} \equiv e^{2 \pi i \nu}
$$

stemming from the simultaneous geometric rotation by $e^{2 \pi i}$ on the $\Psi_{i} \equiv a, b, c, d \in \mathbb{C}^{4}$, recast in terms of the $z_{i}$. The phases $e^{2 \pi i / n_{k}}$ induced on the $z_{i}$ by the independent rotations on the underlying variables $a, b, c, d$, determine the geometry of deformations of the conifold singularity itself. These induce a quotient structure on the flip region with a discrete group $\Gamma$ (analogous 


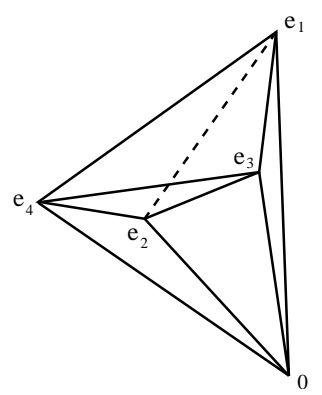

Figure 2: The toric fan for a flip region alongwith the two small resolutions.

to the $\mathbb{Z}_{6}$ earlier): the coordinates $z_{i}$ have the identifications

$$
\begin{aligned}
\left(\begin{array}{rlll}
z_{1} & z_{2} & z_{3} & z_{4}
\end{array}\right) & \longrightarrow^{a}\left(\begin{array}{llll}
e^{2 \pi i / n_{1}} z_{1} & e^{2 \pi i / n_{1}} z_{2} & z_{3} & z_{4}
\end{array}\right) \\
& \longrightarrow^{b}\left(\begin{array}{llll}
z_{1} & z_{2} & e^{2 \pi i / n_{2}} z_{3} & e^{2 \pi i / n_{2}} z_{4}
\end{array}\right), \\
& \longrightarrow^{c}\left(\begin{array}{llll}
e^{2 \pi i / n_{3}} z_{1} & z_{2} & e^{2 \pi i / n_{3}} z_{3} & z_{4}
\end{array}\right) \\
& \longrightarrow^{d}\left(\begin{array}{llll}
z_{1} & e^{2 \pi i / n_{4}} z_{2} & z_{3} & e^{2 \pi i / n_{4}} z_{4}
\end{array}\right)
\end{aligned}
$$

under the independent rotations on each of $a, b, c, d$. If the $n_{i}$ are coprime, then this orbifold action can be described by a cyclic group $\mathbb{Z}_{N}=\prod_{i} \mathbb{Z}_{n_{i}}$, where $N=\operatorname{lcm}\left(n_{1}, n_{2}, n_{3}, n_{4}\right)$ is the least common multiple of the $n_{i}$. Thus in general the flip conifold $\mathcal{C}^{(\text {flip })}$ described by $Q=\left(\begin{array}{llll}n_{1} & n_{2} & -n_{3} & -n_{4}\end{array}\right)$ is the quotient ${ }^{4}$

$$
\mathcal{C}^{(\text {flip })}=\frac{\mathcal{C}}{\prod_{i} \mathbb{Z}_{n_{i}}}
$$

of the supersymmetric conifold $\mathcal{C}$ with the action given by (23), and can be described as the hypersurface (21) in $\mathbb{C}^{4} / \Gamma$ where the discrete group acts as (23) on the coordinates $z_{i}$. With the identifications (23), the relation (24) is one-to-one and the inverse is single-valued. Locally the space is isomorphic to the supersymmetric conifold. Note that if the $z_{i}=0$, then we have $a, b, c, d=0$. If say $z_{1} \neq 0$, then, as in Sec. 5.5 of [12], we can take $a \neq 0$ without loss of

\footnotetext{
${ }^{4}$ See e.g. [33] for a description of this sort of quotient map in the context of Calabi-Yau spaces defined in weighted projective spaces. In particular a weighted $\mathbb{P}_{\mathbf{w}}^{n}\left(x_{0}: \ldots: x_{n}\right)$ with the $x_{i}$ satisfying $\left(x_{0}: \ldots: x_{n}\right) \rightarrow$ $\left(\lambda^{w_{0}} x_{0}: \ldots: \lambda^{w_{n}} x_{n}\right)$, can be described as the quotient

$$
\mathbb{P}_{\mathbf{w}}^{n}=\frac{\mathbb{P}^{n}}{\prod_{i} \mathbb{Z}_{w_{i}}}
$$

of the "isotropic" $\mathbb{P}^{n}\left(z_{0}: \ldots: z_{n}\right)$ having $\mathbf{w}=1$, via the quotient map $x_{i}=z_{i}^{w_{i}}$. In order for the map to be one-to-one, i.e. for an inverse to be well-defined, we must require that the coordinates $z_{i}$ have the identifications $z_{i} \rightarrow e^{2 \pi i / w_{i}} z_{i}$.
} 
generality, and then iteratively solve (20) for $c, d, b$, as

$$
c=\frac{z_{1}^{n_{3}}}{a^{n_{3} / n_{1}}}, \quad d=\frac{z_{2}^{n_{4}}}{a^{n_{4} / n_{1}}}, \quad b=\frac{z_{3}^{n_{2}}}{c^{n_{2} / n_{3}}} .
$$

Note that this is a well-defined inverse map, incorporating the identifications (23) on the $z_{i}$, as well as the relation (21). This shows that the quotient relation (24) gives a space isomorphic to the toric definition using the charge matrix (18).

It is useful to note that the conifold relation (21) in terms of the coordinates $z_{i}$ subject to orbifolding is defined "upstairs", i.e. on the covering space. One can realize other relations between invariant monomials that are single-valued "downstairs" by using the orbifold action (23) as follows. Define

$$
m_{1}=\frac{N}{\operatorname{lcm}\left(n_{1}, n_{3}\right)}, \quad m_{4}=\frac{N}{\operatorname{lcm}\left(n_{2}, n_{4}\right)}, \quad m_{2}=\frac{N}{\operatorname{lcm}\left(n_{1}, n_{4}\right)}, \quad m_{3}=\frac{N}{\operatorname{lcm}\left(n_{2}, n_{3}\right)},
$$

which are all integers. Then a useful set of invariants defining a polynomial ring on this space can be defined as

$$
s=z_{1}^{N / m_{1}}, \quad t=z_{4}^{N / m_{4}}, \quad u=z_{2}^{N / m_{2}}, \quad v=z_{3}^{N / m_{3}}
$$

satisfying the "downstairs" relation

$$
s^{m_{1}} t^{m_{4}}=u^{m_{2}} v^{m_{3}}
$$

which is essentially the $N$-th power of the conifold equation (21) "upstairs" recast in terms of the invariants (27). In general, we also require other monomials invariant under the orbifold action (23) to describe the variety completely "downstairs". These monomials can also be written as invariants under the original $\mathbb{C}^{*}$ action, in terms of the underlying variables $a, b, c, d$. The toric variety itself is described in terms of the set of relations between these monomials. In general, such spaces are not complete intersections of hypersurfaces, i.e. the number of variables minus the number of equations is not equal to the dimension of the space.

Note that the variety $s^{l} t^{l}=u^{k} v^{k}$ defined by (28) for the case $m_{1}=m_{4}=l, m_{2}=m_{3}=k$, obtained by e.g. $n_{1}=n_{2}=k, n_{3}=n_{4}=l$ is on a different footing from similar varieties (and their brane duals) studied in e.g. 34 35] 36. The latter obtain these varieties as supersymmetric quotients of the supersymmetric conifold (where the underlying variables $a, b, c, d$ have charges $n_{i}= \pm 1$ with $\left.\sum Q_{i}=0\right)$. In our case on the other hand, the underlying geometry defined by the variables $a, b, c, d$ has nontrivial dynamics since it corresponds to a charge matrix of the form $Q=\left(\begin{array}{llll}k & k & -l & -l\end{array}\right)$ with $\sum Q_{i}=2(k-l) \neq 0$. The dynamics here is therefore that of an unstable geometry as we will see later in Sec. 3.3 using linear sigma models. 
Examples: Consider the flip region $Q=\left(\begin{array}{llll}1 & 2 & -1 & -3\end{array}\right)$ of the previous section: here the discrete group $\Gamma=\mathbb{Z}_{2} \times \mathbb{Z}_{3}$ is cyclic, giving $\Gamma=\mathbb{Z}_{6}$ and (23) effectively reduces to $\left(\begin{array}{llll}z_{1} & z_{2} & z_{3} & z_{4}\end{array}\right) \rightarrow\left(\begin{array}{cccc}z_{1} & \omega^{2} z_{2} & \omega^{3} z_{3} & \omega^{5} z_{4}\end{array}\right), \omega=e^{2 \pi i / 6}$. Then we have $\left(m_{1}, m_{2}, m_{3}, m_{4}\right)=$ $(6,2,3,1)$ and the monomial invariants $s=z_{1}, t=z_{4}^{6}, u=z_{2}^{3}, v=z_{3}^{2}, w=z_{2} z_{4}^{2}, x=z_{3} z_{4}^{3}$, with the relations $s^{6} t=u^{2} v^{3}, w^{3}=u v, x^{2}=t v$, st $=w x$. In terms of the original $\mathbb{C}^{*}$ action, these monomials can be written as $s=a c, t=b^{3} d^{2}, u=a^{3} d, v=b c^{2}, w=a b d, x=b^{2} c d$, with the relations above.

For the singularity $Q=\left(\begin{array}{cccc}1 & n & -1 & -n\end{array}\right)$, the discrete group is $\mathbb{Z}_{n} \times \mathbb{Z}_{n}$. In this case, we obtain $s=z_{1}, t=z_{4}^{n}, u=z_{2}^{n}, v=z_{3}^{n}$, satisfying $s^{n} t=u v$. Alternatively from the original $\mathbb{C}^{*}$ action, we have $s=a c, t=b d, u=a^{n} d, v=b c^{n}$.

\subsubsection{On deformations of the singularity}

In this section, we want to look for possible 3-cycle $\epsilon$-deformations of the form

$$
z_{1} z_{4}-z_{2} z_{3}=\epsilon
$$

for flip conifolds $\left(\sum Q_{i} \neq 0\right)$ with the hypersurface equation (21), with $\epsilon$ chosen a real parameter (by coordinate changes). Since the flip conifold is a quotient of the supersymmetric conifold (described as a 3-complex dimensional hypersurface embedded in the orbifold $\mathbb{C}^{4} / \Gamma$ with the conifold singularity coinciding with the orbifold singularity), one might imagine that such deformations of the hypersurface singularity are obstructed by the quotient structure, drawing intuition from the fact that toric $\mathbb{C}^{d} / G, d>2$, orbifolds themselves do not admit any such complex structure deformations [37]. Following this logic shows that this is in fact true whenever the singularity is isolated, i.e. the $n_{i}$ are all coprime ${ }^{5}$ in which case $\Gamma$ is a cyclic group. Further analysis using the symmetries of the underlying variables $a, b, c, d$, or equivalently the structure (24) of these singularities as quotients of the supersymmetric conifold, shows that in fact 3-cycle deformations are always obstructed, as we will see below. Another way to state this is: the only complex structure deformation of the supersymmetric conifold is of the form (29), and the quotient structure of the flip conifold imposes global obstructions to this possible local deformation 6 . In what follows, we continue to use the phrase "3-cycle" deformations rather than complex structure deformations, since we are really only focussing on the local geometry in the vicinity of the singularity, rather than the full compact embedding space which is where one would conventionally define Kähler and complex structure deformations.

\footnotetext{
${ }^{5}$ The toric fan of the singularity encodes information such as whether it is isolated, as we describe in the next section.

${ }^{6}$ This does not however preclude abstract deformations: see later.
} 
To begin, let us study flip regions in terms of $\nu$, the integers $n_{i}$ in $Q$ being an ordered set. By definition, we have $0 \leq \nu \leq 4 . \nu=4$ is only possible when all $n_{i}=1$ (this is the supersymmetric conifold), while $\nu=0$ is only possible for all $n_{i} \rightarrow \infty$. Thus any finite $n_{i}$ can at best give $\nu=1,2,3$ for integral $\nu$. From the action of the discrete group (23) on the coordinates (20) and the structure of the conifold equation (21), we see that whenever $\nu$ is not integral, the phase $e^{2 \pi i \nu}$ given in (22) acting on each of the two terms of the conifold equation is nontrivial, so that the $\epsilon$-deformation is obstructed. In fact it is straightforward to show that whenever the singularity is isolated, i.e. the $n_{i}$ are coprime w.r.t. each other, the phase $e^{2 \pi i \nu}$ is nontrivial and the deformation is obstructed (see the Appendix).

Now if $\nu \in \mathbb{Z}$ as in the select non-isolated cases described in the Appendix, then the phase $e^{2 \pi i \nu}$ is trivial: this would suggest that there is no obstruction to the 3-cycle $\epsilon$-deformation of the flip conifold equation (21). If the phases induced by rotations in $a, b, c, d$, were correlated, then the embedding orbifold group $G$ would be cyclic, satisfying $\operatorname{det} G=e^{2 \pi i(2 \nu)}=1$, in other words, the singularity would be embedded in a supersymmetric $\mathbb{C}^{4} / G$ orbifold, with the same holonomy $(S U(4))$ as a Calabi-Yau 4-fold. For such cases, the analysis of possible 3 -cycles would coincide mathematically with the analysis of [39] who study $S^{3} / G$ in a supersymmetric context. The 3 -cycle in these cases is the locus $z_{4}=z_{1}^{*}, z_{3}=-z_{2}^{*}$, with some discrete identifications imposed by the orbifolding, giving $S^{3} / G$ rather than $S^{3}$, the orbifold $G$ acting freely on the $S^{3}$. For example, consider the conifold singularity with the identifications $\left(\begin{array}{llll}z_{1} & z_{2} & z_{3} & z_{4}\end{array}\right) \rightarrow\left(\begin{array}{llll}-z_{1} & -z_{2} & -z_{3} & -z_{4}\end{array}\right)$. Define new coordinates $w_{i}$ in terms of linear combinations $z_{1}=w_{1}+i w_{4}$ etc, so that the deformed singularity (29) is now written as $\sum_{i=1}^{4} w_{i}^{2}=\epsilon$, with the 3 -cycle being the locus where the $w_{i}$ are real. The identifications on the $z_{i}$ translate to the identifications $w_{i} \rightarrow-w_{i}$, giving $S^{3} / \mathbb{Z}_{2}$ here. This leads one to ask what the corresponding structure of possible deformed singularities is (if they exist), in the case when the orbifold group is not cyclic.

With this in mind, recall that the phase rotations of the underlying variables $a, b, c, d$, defining the geometric space are independent. These are basically the residual, i.e. unquotiented, torus actions that were present in the toric variety defined by the underlying variables $a, b, c, d$ (and in the corresponding linear sigma model of the next section). Thus consider the path (starting and ending at the same point on the space) given by

$$
d \rightarrow e^{2 \pi i} d, \quad a, b, c=\text { fixed } .
$$

Such paths define isometry directions of the geometry, so the deformed hypersurface equation should also respect these if the deformation is consistent. However from (20) (23), we see that only $z_{2}, z_{4}$ acquire a phase and the conifold equation (29) transforms as

$$
\epsilon=z_{1} z_{4}-z_{2} z_{3} \rightarrow e^{2 \pi i / n_{4}}\left(z_{1} z_{4}-z_{2} z_{3}\right)
$$


and similarly for any other such path. For $n_{i} \neq 1$, this is consistent only if $\epsilon=0$, i.e. the deformation is obstructed. Another way of seeing that this is sensible is to realize that a holomorphic 3-form on the deformed side does not exist either, being projected out by the above phase.

The arguments here have used the "upstairs" variables, carefully implementing the toric symmetries of the underlying geometry described by the variables $a, b, c, d$ : note that these toric symmetries are equivalent to the structure (23) (24) of these singularities as quotients of the supersymmetric conifold. In other words, the expression (29) is not invariant under the identifications (23) defining the relation (24), unless the deformation parameter $\epsilon=0$. These arguments are reasonable for deformations connected to the singular point since infinitesimal deformations that can be interpreted "upstairs" (i.e. in the supersymmetric conifold) must be consistent with the additional toric symmetries (or equivalently the quotient symmetries) present for flips. It is noteworthy that these quotient (or equivalently toric) symmetries thus obstruct the only complex structure deformation (which is not toric) of the supersymmetric conifold.

Now we make a few remarks on abstract deformations of these singularities which may not allow any interpretation in terms of the "upstairs" structure. It appears that the space of toric deformations (characterized by the fact that the total space including the deformation still belongs to the toric category) is a large subset of the so-called versal, or complete, deformation of a singularity (see e.g. 40, for deformations of Gorenstein singularities ${ }^{7}$ ). The versal deformation for a space described as the variety $f\left(x_{1}, \ldots, x_{n}\right)=0$, is given as $\mathbb{C}\left[x_{1}, \ldots, x_{n}\right] /\left(f, \partial f / \partial x_{1}, \ldots, \partial f / \partial x_{n}\right)$, i.e. the polynomial ring modulo the ideals given by the vanishing of $f$ and its first partial derivatives $f_{i} \equiv \partial f / \partial x_{i}$ : this is reasonable to see for infinitesimal deformations since a coordinate transformation $x_{i}^{\prime}=x+\delta x_{i}$, of the $x_{i}$ can absorb first order changes as $f\left(x_{i}\right)=f\left(x_{i}^{\prime}\right)-f_{i} \delta x_{i}$. It is in general not easy to calculate the versal deformation of singularities outside of the formal techniques of e.g. [37, 40]. However we can get some intuition for this in simple examples. In terms of coordinates well-defined "downstairs", we can describe e.g. the simplest flip singularity $Q=\left(\begin{array}{llll}1 & 1 & -1 & -2\end{array}\right)$ as follows. The "upstairs" coordinates $z_{i}$ have the $\mathbb{Z}_{2}$ identifications $\left(\begin{array}{llll}z_{1} & z_{2} & z_{3} & z_{4}\end{array}\right) \rightarrow^{d}\left(\begin{array}{cccc}z_{1} & -z_{2} & z_{3} & -z_{4}\end{array}\right)$. The "downstairs" coordinates $s=z_{1}=a c, t=z_{2}^{2}=a^{2} d, u=z_{3}=b c, v=z_{4}^{2}=b^{2} d, w=z_{2} z_{4}$, generating the monomial ring on the space, satisfy the relations $f_{1}=t v-w^{2}=0, f_{2}=s v-u w=0, f_{3}=$ $t u-s w=0$. This set at quadratic order can be regarded as a basis for the ideal of relations in this case, since higher order relations follow from these. Note that this set of three relations in $\mathbb{C}^{5}[s, t, u, v, w]$ is not a complete intersection: the toric variety defined by $a, b, c, d$, is 3-complex

\footnotetext{
${ }^{7}$ See also e.g. [41] for semi-universal deformations of ground varieties in the context of $c=1$ string theory.
} 
dimensional, which can also be seen by studying the rank of the derivative matrix $\left[\frac{\partial f_{i}}{\partial x_{j}}\right]$. Alternatively on the coordinate patch with nonzero $s, t, u$, we have $w=\frac{t u}{s}, v=\frac{t u^{2}}{s^{2}}$ (the third potential relation being an identity), so that the space is described by the variables $s, t, u$ (similarly for other charts). Thus this singularity is embedded in $\mathbb{C}^{5}[s, t, u, v, w]$ as the intersection of the supersymmetric conifolds $s v-u w=0$ and $s w=t u$, with the $\mathbb{C}^{2} / \mathbb{Z}_{2}$ orbifold $t v-w^{2}=0$. Let us look for deformations of the two conifolds consistent with a deformation of the orbifold: specifically consider $f_{1}^{\prime}=t v-w^{2}-\epsilon=0, f_{2}^{\prime}=s v-u w-\lambda=0, f_{3}^{\prime}=s w-t u-\mu=0$, with $\epsilon, \lambda, \mu$ being real. On the $(s, t, u)$ patch, the 3 -cycle in $f_{2}^{\prime}$ is the locus $v=s^{*}, w=-u^{*}$, while that in $f_{3}^{\prime}$ is $w=s^{*}, u=-t^{*}$, i.e. we have $s=v^{*}=w^{*}=-u=t^{*}$ : this gives $\epsilon=\left(s^{*}\right)^{2}-\left(s^{*}\right)^{2}=0$ (from $f_{1}^{\prime}$ ), and $\lambda=\mu=2 s s^{*}$, which is a $\mathbb{P}^{1}$. This abstract deformation (which is not a 3 -cycle topologically) is difficult to interpret "upstairs" in terms of the quotient structure: the total space in this case is not toric. For the general singularity (18), writing the explicit monomial relations and visualizing the geometry is hard, which also complicates the set of such abstract deformations even along the lines of this crude analysis in this simple example. It is also interesting to understand the constraints of unbroken worldsheet supersymmetry on the quantum descriptions of these 2D theories besides the obstructions obtained from the classical geometry analysis here (see Sec. 3.3 and the Discussion). The analysis here is of course purely within geometry, not accounting for any intrinsically stringy branch e.g. along some flux direction.

To summarize we have looked for 3-cycle deformations using the quotient structure (24) (23): : in principle there could exist abstract deformations of the singularity lying "outside" this structure, invisible to the techniques used here. It would be interesting to understand this better, perhaps using the formal techniques of [37, 40].

\subsection{Small resolutions and their dynamics}

Let us now study the dynamics of the small resolutions: these necessarily have an inherent directionality whenever $\sum Q_{i} \neq 0$, as we will see from a linear sigma model analysis below. Consider a basis for the $\boldsymbol{N}$ lattice (and the toric cone in it) given by

$$
e_{2} \equiv \phi_{2}=(1,0,0), \quad e_{3} \equiv \phi_{3}=(0,1,0), \quad e_{4} \equiv \phi_{4}=(0,0,1)
$$

Then the fourth vertex $\phi_{1}$ of the cone (see Figure 21) in $\boldsymbol{N}$ defining the conifold (see (19)) can be rewritten in terms of the $e_{1}, e_{2}, e_{3}$ basis as

$$
e_{1} \equiv \phi_{1}=-\frac{n_{2}}{n_{1}} e_{2}+\frac{n_{3}}{n_{1}} e_{3}+\frac{n_{4}}{n_{1}} e_{4} \longrightarrow_{n_{1}=1}\left(-n_{2}, n_{3}, n_{4}\right) \text {. }
$$

(Despite appearances for $n_{1} \neq 1$, the lattice here is integral as we clarify below.) Whenever the $n_{i}$ are relatively coprime, this toric fan does not contain any lattice points in its interior 
or on its "walls" - this is an isolated singularity (see the Example at the end of this Section). The four vertices $\left\{e_{1}, e_{2}, e_{3}, e_{4}\right\}$ are coplanar, lying on a hyperplane, if

$$
0=\operatorname{det}\left(e_{1}-e_{2}, e_{3}-e_{2}, e_{4}-e_{2}\right)=\frac{n_{3}+n_{4}-n_{1}-n_{2}}{n_{1}},
$$

in other words, $\sum Q_{i}=\Delta n=0$. In this case, the two small resolutions give identical residual volumes, so that there is no intrinsic directionality to their dynamics: the fan corresponds to a spacetime supersymmetric geometry and the two resolutions are related by a flop, i.e. a marginal deformation. On the other hand, non-coplanarity of the $\left\{e_{i}\right\}$ means that the two small resolutions (related by a flip transition) give distinct residual subcone volumes for their corresponding partial blowups, so that one expects an inherent directionality in the dynamics of the geometry. In both cases, the two small resolutions are topologically distinct since when this geometry is embedded in a compact space, the intersection numbers of various cycles change under the flip/flop.

We can calculate the subcone volumes for each of the small resolutions (see Figure 2):

$$
\begin{aligned}
& \mathbb{P}_{+}^{1}: V_{+}=V\left(0 ; e_{1}, e_{2}, e_{3}\right)+V\left(0 ; e_{1}, e_{2}, e_{4}\right)=\frac{n_{4}}{n_{1}}+\frac{n_{3}}{n_{1}} \\
& \mathbb{P}_{-}^{1}: V_{-}=V\left(0 ; e_{2}, e_{3}, e_{4}\right)+V\left(0 ; e_{1}, e_{3}, e_{4}\right)=1+\frac{n_{2}}{n_{1}}
\end{aligned}
$$

so that the difference in volumes is

$$
\Delta V=V_{-}-V_{+}=\frac{\Delta n}{n_{1}} .
$$

This represents the difference in the cumulative degrees of the residual singularities for the two small resolutions, and is non-vanishing if $\sum_{i} Q_{i}=\Delta n \neq 0$. Note that in the normalization where the supersymmetric conifold has the charge matrix (14), i.e. $Q=\left(\begin{array}{llll}1 & 1-1 & -1\end{array}\right)$, we have its residual cumulative volumes $V_{ \pm}=2$, so that any singularity with $n_{1} \neq 1$ potentially has fractional volumes $V_{ \pm}$- in the latter cases, one can choose a different normalization for the supersymmetric conifold, which then yields an integral $\boldsymbol{N}$ lattice. For simplicity, let us set $n_{1}=1$ to obtain an integral lattice for arbitrary $n_{2}, n_{3}, n_{4}$ : this gives $\Delta V=\Delta n$. Then the small resolution decay modes of the flip conifold give rise to the four residual subcones $C\left(0 ; e_{i}, e_{j}, e_{k}\right)$ which are potentially $\mathbb{C}^{3} / \mathbb{Z}_{M}$ singularities. For all Type II theories, these have either moduli or tachyons in their spectrum of deformations which can resolve them completely. Recalling that $\mathbb{C}^{3} / \mathbb{Z}_{2}(1,1,1)$ is a truly terminal singularity appearing in the spectrum of decay endpoints in Type 0 unstable orbifolds [8], we obtain a constraint on when these flip conifolds decay to smooth spaces assuming the GSO projection is preserved along the flow as it is in orbifolds [8]. For example, $C\left(0 ; e_{1}, e_{3}, e_{4}\right)$ corresponds to $\mathbb{Z}_{n_{2}}\left(1,-n_{3},-n_{4}\right)$, so that if $n_{2}=2$, we must have 
at least one of $n_{3}, n_{4}$ to be even for this subcone to not be $\mathbb{C}^{3} / \mathbb{Z}_{2}(1,1,1$ ) (for a Type II theory, this condition is automatically met since the GSO projection [8] requires $n_{3}+n_{4}=$ odd here). This is a nontrivial requirement: for instance, the singularity $Q=\left(\begin{array}{llll}1 & 2 & -1 & -3\end{array}\right)$ discussed earlier does not satisfy this and in fact exhibits the terminal singularity in its decay endpoints.

In fact we can obtain a general constraint on the $n_{i}$ from the known Type II GSO projection $\sum k_{i}=$ even [8] on the $\mathbb{C}^{3} / \mathbb{Z}_{M}\left(k_{1}, k_{2}, k_{3}\right)$ decay endpoints at the IR, if we assume that a GSO projection defined in the UV is not broken along the RG flow corresponding to the decay. From the Smith normal form algorithm of [8] (or otherwise), we can see that the various residual subcones correspond to the orbifolds $C\left(0 ; e_{1}, e_{2}, e_{3}\right) \equiv \mathbb{Z}_{n_{4}}\left(1, n_{2},-n_{3}\right), C\left(0 ; e_{1}, e_{2}, e_{4}\right) \equiv$ $\mathbb{Z}_{n_{3}}\left(1, n_{2},-n_{4}\right)$, and $C\left(0 ; e_{1}, e_{3}, e_{4}\right) \equiv \mathbb{Z}_{n_{2}}\left(1,-n_{3},-n_{4}\right)$, up to shifts of the orbifold weights by the respective orbifold orders, since these cannot be determined unambiguously by the Smith algorithm $^{8}$. Then we can see that each of these orbifolds in the IR of the conifold decay admit a consistent Type II GSO projection if

$$
\Delta n=\sum Q_{i}=n_{1}+n_{2}-n_{3}-n_{4}=\text { even }
$$

If this condition is satisfied, string theory in the flip conifold spacetime background in question does not have a bulk tachyon at the top of the tachyon "hill". Setting $n_{1}=1$ for simplicity again, this implies that $n_{2}+n_{3}+n_{4}=o d d$, since $\Delta n$ in (37) is only defined mod 2. To illustrate this, consider, without loss of generality, the case $n_{2}=$ even. Then we must have that $n_{3}+n_{4}=$ odd, i.e. one and only one of $n_{3}, n_{4}$ is odd. This means that $C\left(0 ; e_{1}, e_{3}, e_{4}\right) \equiv$ $\mathbb{Z}_{n_{2}}\left(1,-n_{3},-n_{4}\right)$ automatically admits a Type II GSO projection. Now say $n_{3}=$ odd. Then the subcone $C\left(0 ; e_{1}, e_{2}, e_{3}\right) \equiv \mathbb{Z}_{n_{4}}\left(1, n_{2},-n_{3}\right)$ also admits a Type II GSO projection, while the subcone $C\left(0 ; e_{1}, e_{2}, e_{4}\right) \equiv \mathbb{Z}_{n_{3}}\left(1, n_{2},-n_{4} \pm n_{3}\right)$, after shifting one of the weights by the order $n_{3}$, is also seen to admit a Type II GSO projection. It is straightforward to show that the other cases are similarly dealt with. For instance, we can see from this condition that the singularity $Q=\left(\begin{array}{llll}1 & 2 & -1 & -3\end{array}\right)$ cannot be Type II, consistent with finding the terminal singularity in its decay endpoints.

Now consider the dynamics of the small resolutions described by the $U(1)$ gauged linear sigma model with the four scalars $\Psi \equiv a, b, c, d$, and a Fayet-Iliopoulos (real) parameter $r$ governing the vacuum structure: this system has $(2,2)$ worldsheet supersymmetry. The fields $\Psi$ transform under $U(1)$ gauge transformations with the charge matrix $Q_{i}$ as

$$
\Psi_{i} \rightarrow e^{i Q_{i} \beta} \Psi_{i}, \quad Q_{i}=\left(n_{1}, n_{2},-n_{3},-n_{4}\right)
$$

$\beta$ being the gauge parameter. As in the description of orbifold flips in Sec. 2, the action for

\footnotetext{
${ }^{8}$ It is useful to note that these $\mathbb{C}^{3} / \mathbb{Z}_{M}$ orbifolds in the IR are isolated if the $n_{i}$ are all relatively coprime.
} 
the GLSM is

$$
S=\int d^{2} z\left[d^{4} \theta\left(\bar{\Psi}_{i} e^{2 Q_{i} V} \Psi_{i}-\frac{1}{4 e^{2}} \bar{\Sigma} \Sigma\right)+\operatorname{Re}\left(i t \int d^{2} \tilde{\theta} \Sigma\right)\right]
$$

where $t=i r+\frac{\theta}{2 \pi}, \theta$ being the $\theta$-angle in $1+1$-dimensions. The classical vacuum structure can be found, as in Sec. 2, by the bosonic potential (4): as in that case, the $\sigma$ fields have zero vevs for nonzero $r$, and the classical vacuum structure is described by the D-term equation

$$
-\frac{D}{e^{2}}=n_{1}|a|^{2}+n_{2}|b|^{2}-n_{3}|c|^{2}-n_{4}|d|^{2}-r=0
$$

divided by $U(1)$, from which one can realize the two small resolutions (rank-2 bundles over $\mathbb{P}_{ \pm}^{1}$ ) as manifested by the moduli space for the single FI parameter ranges $r \gg 0$ and $r \ll 0$. The geometry of this space can be understood thinking of this as a symplectic quotient. Firstly consider the phase $r \gg 0$ : then one of $a, b$ is nonzero. Consider for simplicity the case where $n_{i}$ are all coprime. Then $z_{+}=\frac{a^{n_{2}}}{b^{n_{1}}}$, invariant under the $U(1)$ defines a coordinate on the $\mathbb{P}^{1}$ on the patch where $b \neq 0$ (with $z_{-}=\frac{1}{z_{+}}$the corresponding coordinate on the patch where $a \neq 0$ ). The full space, a rank-2 bundle over $\mathbb{P}^{1}$, can then be described in terms of the invariants $p_{-}=a^{n_{3}} c^{n_{1}}, p_{+}=b^{n_{3}} c^{n_{2}}, q_{-}=a^{n_{4}} d^{n_{1}}, q_{+}=b^{n_{4}} d^{n_{2}}$, satisfying the relations $p_{-}^{n_{2}}=p_{+}^{n_{1}} z_{+}^{n_{3}}, q_{-}^{n_{2}}=q_{+}^{n_{1}} z_{+}^{n_{4}}$, which describes the rank-2 bundle locally as a variety embedded in $\mathbb{C}^{2}\left(p_{-}, p_{+}\right) \times \mathbb{C}^{2}\left(q_{-}, q_{+}\right)$. This is the small resolution for the phase $r \gg 0$. The residual $\mathbb{C}^{3} / \mathbb{Z}_{M}$ singularities in this phase are realized by looking at the regions in moduli space where only one of $a, b$, acquires a vacuum expectation value: for instance, a vev for $b$ alone Higgses the $U(1)$ down to $\mathbb{Z}_{n_{2}}$ with the chart $(a, c, d)$, i.e. the subcone $C\left(0 ; e_{1}, e_{3}, e_{4}\right)$.

The other small resolution for the phase $r \ll 0$ can be similarly studied in terms of the invariant coordinates $z_{+}^{\prime}=\frac{c^{n_{4}}}{d^{n_{3}}} \equiv \mathbb{P}^{1}$, and $p_{-}^{\prime}=c^{n_{1}} a^{n_{3}}, p_{+}^{\prime}=d^{n_{1}} a^{n_{4}}, q_{-}^{\prime}=c^{n_{2}} b^{n_{3}}, q_{+}=d^{n_{2}} b^{n_{4}}$, satisfying the relations $p_{-}^{\prime n_{4}}={p_{+}^{\prime}}^{n_{3}} z_{+}^{\prime n_{1}}, q_{-}^{\prime n_{4}}=q_{+}^{\prime n_{3}} z_{+}^{\prime n_{2}}$, which describes the bundle locally as a variety in $\mathbb{C}^{2}\left(p_{-}^{\prime}, p_{+}^{\prime}\right) \times \mathbb{C}^{2}\left(q_{-}^{\prime}, q_{+}^{\prime}\right)$.

This describes how the resolved flip conifold phases, rank-2 bundles over $\mathbb{P}_{ \pm}^{1}$, are embedded in general locally as 3-complex dimensional spaces in $\mathbb{P}_{ \pm}^{1} \times \mathbb{C}^{2} \times \mathbb{C}^{2}$. Note that for special cases, this simplifies to the structure of an $\mathcal{O}\left(-l_{1}\right) \oplus \mathcal{O}\left(-l_{2}\right)$ bundle over $\mathbb{P}^{1}$, for appropriate integers $l_{1}, l_{2}$, as we will see in an example later. Perhaps one can also usefully describe the total space in general as a bundle analog of an orbifold since the fibre coordinates $p_{ \pm}, q_{ \pm}$(as well as $\left.p_{ \pm}^{\prime}, q_{ \pm}^{\prime}\right)$ above have identifications. For the case where the $n_{i}$ are not all coprime, one defines coordinates on the $\mathbb{P}^{1}$ after eliminating the g.c.d. and so on, and similarly for the fibre coordinates.

Analogous to (11) for orbifolds, the parameter $r$ has a 1-loop renormalization given by

$$
r=\left(\sum_{i} Q_{i}\right) \log \frac{\mu}{\Lambda}=(\Delta n) \log \frac{\mu}{\Lambda}=(\Delta V) \log \frac{\mu}{\Lambda}
$$


(setting $n_{1}=1$ here for simplicity) identifying the coefficient of the logarithm with the volume difference (36). For $\sum_{i} Q_{i}=0$, there is no 1-loop renormalization and $r$ is expected to correspond to a marginal operator in the corresponding string conformal field theory. Note that the two phases are still topologically distinct geometries in general, as described above. For $\sum_{i} Q_{i} \neq 0$, the GLSM RG flow (41) drives the system towards the phase corresponding to smaller $\boldsymbol{N}$ lattice volume, the residual volumes for the two resolutions and their differences being given in (35) (36), much like the corresponding flow (10) (11) in orbifold flips described in Sec. 2. This shows that the conifold dynamically evolves towards the less singular, and therefore more stable, small resolution, similar to flips arising in unstable orbifolds [9]. In particular, if one sets up initial conditions for the geometry to lie in the less stable small resolution, then small fluctuations will force the system to evolve through a flip transition: the mild topology change here, with the blown-down 2-cycle $\mathbb{P}_{+}^{1}$ and the blown-up 2-cycle $\mathbb{P}_{-}^{1}$ changing the intersection numbers ${ }^{9}$ of various cycles, is dynamically mediated by closed string tachyon instabilities in the geometry. Our discussion here is restricted to the large $r$ semiclassical regions of the geometry where quantum corrections are small, but this is sufficient insofar as an understanding of the long $(\mathrm{RG})$ timescale dynamics of the system is concerned. It is important to note that we have decoupled gravity here, as for orbifold flips in Sec. 2: this assumption on the validity of the GLSM for long timescales is equivalent to the reasonable assumption that operator mixing along RG trajectories is negligible insofar as an understanding of the phase structure at the onset of localized tachyon condensation is concerned (the GSO projection being preserved is a consistency check). From a spacetime point of view, operator mixing is expected on sufficiently long timescales where one must pass to an appropriate gravity description.

We can calculate the bosonic potential (13) from the quantum twisted chiral superpotential (12) as

$$
U(\sigma)=\frac{e^{2}}{2}\left|i \hat{\tau}-\frac{\sum_{i} Q_{i}}{2 \pi}\left(\log \left(\sqrt{2} Q_{i} \sigma / \Lambda\right)+1\right)\right|^{2} .
$$

As for orbifold flips [9], the potential has a $|\log \sigma|^{2}$ growth along the relevant direction which is the single direction here: thus the quantum corrections serve to smoothing out the transition avoiding the classical singularity at $r=0$. It would appear that there exist isolated Coulomb branch $\sigma$-vacua in the infrared of this system too, as in unstable orbifolds: perhaps the analysis there [6, 17, 13, 15, 16] can also be used to give insight here ${ }^{10}$.

Note that we have not actually constructed the tachyonic states explicitly in a conformal

\footnotetext{
${ }^{9}$ The changes in e.g. the triple intersection numbers of divisors can be seen directly from the toric fan in Figure 2 using standard toric geometry calculations.

${ }^{10}$ Note also the analysis 38 of fractional branes in a nonsupersymmetric $\mathbb{C}^{2} / \mathbb{Z}_{M}$ orbifold via an embedding thereof in a higher dimensional supersymmetric singularity $\mathbb{C}^{3} / \Gamma$. See also [10].
} 
field theory in this discussion: we have resorted to indirect means such as the linear sigma model $^{11}$. It would be interesting to construct e.g. Gepner-like models describing nonsupersymmetric orbifolds of Calabi-Yau spaces which develop flip conifold singularities, with a view to more concretely realizing closed string tachyons in such geometries.

Finally, our assumption that the GSO projection is not broken along the RG flow corresponding to the decay is not unreasonable physically. For instance consider the case when the GSO is broken, i.e. the flip conifold background $\mathbb{R}^{3,1} \times \mathcal{C}^{(\text {flip })}$ has a bulk tachyon while the $\mathbb{C}^{3} / \mathbb{Z}_{M}$ orbifold endpoints in the IR do not. Then along the localized tachyon RG flow described by the GLSM, the bulk tachyon must somehow disappear. This seems unlikely since in spacetime, the decay of this conifold is via a small resolution involving a $\mathbb{P}^{1}$ expanding to large size with potential orbifold singularities whose spatial separation from each other grows at the rate the volume of the 2 -sphere grows. It would seem inconsistent to have a delocalized tachyon suddenly disappear (or appear) in the process of condensation of localized tachyons (which is rendered further credence by the fact that $(2,2)$ worldsheet supersymmetry is not broken in this GLSM): on the other hand, a more conservative view is that the GLSM is perhaps not a good tool to describe tachyon condensation phenomena where the GSO projection is broken. In any case, it would be interesting to recover the GSO constraint (37) more directly, e.g. modular invariance of the partition function of an appropriate Gepner-like model.

Example: Consider the singularity $Q=\left(\begin{array}{llll}1 & 1 & -2 & -2\end{array}\right)$. The coordinates $z_{i}$ have the $\mathbb{Z}_{2} \times \mathbb{Z}_{2}$ identifications $\left(\begin{array}{cccc}z_{1} & z_{2} & z_{3} & z_{4}\end{array}\right) \rightarrow^{c}\left(\begin{array}{cccc}-z_{1} & z_{2} & -z_{3} & z_{4}\end{array}\right)$ and $\left(\begin{array}{llll}z_{1} & z_{2} & z_{3} & z_{4}\end{array}\right) \rightarrow^{d}$ $\left(\begin{array}{llll}z_{1} & -z_{2} & z_{3} & -z_{4}\end{array}\right)$. The "downstairs" coordinates $x_{1}=z_{1}^{2}=a^{2} c, x_{2}=z_{2}^{2}=a^{2} d, x_{3}=z_{3}^{2}=$ $b^{2} c, x_{4}=z_{4}^{2}=b^{2} d, x_{5}=z_{1} z_{3}=a b c, x_{6}=z_{2} z_{4}=a b d$, generating the monomial ring describe the singularity as the intersection of the four relations $x_{1} x_{4}=x_{2} x_{3}, x_{5} x_{6}=x_{1} x_{4}, x_{5}^{2}=$ $x_{1} x_{3}, x_{6}^{2}=x_{2} x_{4}$, in $\mathbb{C}^{6}\left[x_{1}, \ldots, x_{6}\right]$.

This singularity admits a Type II GSO projection $\left(\sum Q_{i}=\right.$ even). From (32) (33), we see that the cone (Figure 2) is defined by the lattice points $e_{2}, e_{3}, e_{4}$ and $e_{1}=(-1,2,2)$. Then $V_{-}=2, V_{+}=4$, and the less singular resolution is $\mathbb{P}_{-}^{1}$. A signature that this is a non-isolated singularity is the interior lattice point $(0,1,1)=\frac{e_{1}+e_{2}}{2}$ lying on the wall $\left\{0, e_{1}, e_{2}\right\}$ defining the $\mathbb{P}_{+}^{1}$ resolution: note that this point lies along $\left\{0, e_{3}, e_{4}\right\}$ defining the less singular $\mathbb{P}_{-}^{1}$ resolution but "above" the cone $\left\{0 ; e_{1}, e_{2}, e_{3}, e_{4}\right\}$. The residual subcones under the resolution $\mathbb{P}_{+}^{1}$ are $C\left(0 ; e_{1}, e_{2}, e_{4}\right) \equiv \mathbb{Z}_{2}(1,1), C\left(0 ; e_{1}, e_{2}, e_{3}\right) \equiv \mathbb{Z}_{2}(1,1)$ (which are both supersymmetric, the point $(0,1,1)$ above defining the marginal blowup mode), with the other two subcones,

\footnotetext{
${ }^{11}$ For flip regions arising in nonsupersymmetric $\mathbb{C}^{3} / \mathbb{Z}_{N}$ orbifolds, one can construct the tachyons explicitly as twisted sector excitations in the orbifold conformal field theory as in [8]
} 
under $\mathbb{P}_{-}^{1}$, being smooth (volume $=1$ ). We have $\sum Q_{i} \neq 0$ here and the GLSM dictates an $\mathrm{RG}$ flow to the $\mathbb{P}_{-}^{1}$ resolution. This corresponds to the total space of an $\mathcal{O}(-2) \oplus \mathcal{O}(-2)$ bundle over $\mathbb{P}_{-}^{1}$, realized by defining $z_{+}=\frac{a}{b}, p_{-}=a^{2} c, p_{+}=b^{2} c, q_{-}=a^{2} d, q_{+}=b^{2} d$, satisfying $p_{-}=p_{+} z_{+}^{2}, q_{-}=q_{+} z_{+}^{2}$. The other resolution is an $\mathcal{O}(-1) \oplus \mathcal{O}(-1)$ bundle over $\mathbb{P}_{+}^{1}$, realized by $z_{+}^{\prime}=\frac{c}{d}, p_{-}^{\prime}=c a^{2}, p_{+}^{\prime}=d a^{2}, q_{-}^{\prime}=c b^{2}, q_{+}^{\prime}=d b^{2}$, satisfying $p_{-}^{\prime}=p_{+}^{\prime} z_{+}^{\prime}, q_{-}^{\prime}=q_{+}^{\prime} z_{+}^{\prime}$.

\section{Discussion}

We have studied the local geometry and dynamics of flip conifold singularities in this work. Along the lines of [42, 32 for the supersymmetric conifold, we can attempt a construction of the quiver theory [43] on a D3-brane probe near a flip conifold singularity. Consider a $U(1) \times U(1)$ gauge theory and bifundamental scalars $A_{1}, A_{2}, B_{1}, B_{2}$ carrying charges $\left(n_{i},-n_{i}\right), i=1 \ldots 4$. These are neutral under the diagonal $U(1)$, the free photon on the D3-brane. The moduli space of vacua is determined by the D-term condition

$$
D=n_{1}\left|A_{1}\right|^{2}+n_{2}\left|A_{2}\right|^{2}-n_{3}\left|B_{1}\right|^{2}-n_{4}\left|B_{2}\right|^{2}-\rho=0
$$

divided by $U(1), \rho$ being the Fayet-Iliopoulos coupling on the D-brane worldvolume. Since $\sum Q_{i} \neq 0$, it is not possible to write a superpotential of the form $\epsilon^{i j} \epsilon^{k l} \operatorname{Tr}\left(A_{i} B_{k} A_{j} B_{l}\right)$ that is invariant under the $U(1)$ action: this is reminiscent of a similar statement for a superpotential like $\operatorname{Tr} X[Y, Z]$ for nonsupersymmetric orbifolds, and is not surprising since spacetime supersymmetry is broken: there would of course be a bosonic potential energy which would be interesting to nail down. We imagine that a stack of $k$ D3-branes sitting at the singularity

(either orbifold or conifold) would admit an $A d S_{5} \times X^{5}$ supergravity description, where $X^{5}$ is some appropriate compact manifold. For isolated singularities, there is no place where light localized tachyonic states can emerge so that one expects a tachyon-free large $k$ (i.e. large flux) description. This suggests that the dual gauge theory is insensitive to the closed string tachyonic instabilities, and thus stable, at leading order in $\frac{1}{k}$. It is tempting to speculate the existence of nontrivial gauge theories by appropriate tuning of the parameters in the geometry and the number $k$ of D3-branes (as well as possible fractional branes), as nonsupersymmetric duals to the supergravity geometries. Subleading $\frac{1}{k}$ effects might then encode the tachyonic instabilities in the gauge theory. It would be interesting to develop this further and understand possible tachyonic open-closed dualities [44].

In general, one imagines such a geometry to be embedded as a local singularity in some compact space: for instance one expects that appropriate nonsupersymmetric orbifolds of Calabi-Yau spaces can be embedding spaces (although perhaps only for the low-lying singularities, i.e. small $n_{i}$ ). We expect that the local analysis here is largely independent of the 


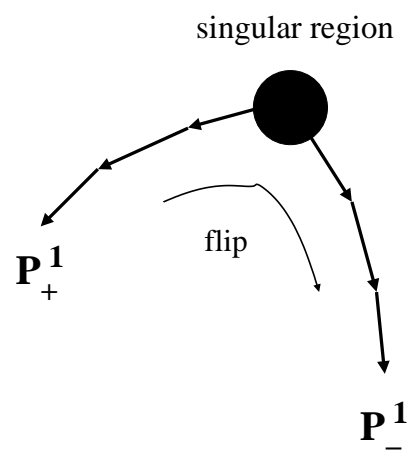

Figure 3: A heuristic picture of the possible directions for the evolution of a flip conifold singularity, the two small resolutions $\mathbb{P}_{ \pm}^{1}$ : possible 3 -cycle deformations are obstructed. The direction of the flip shown here is for the case when $\mathbb{P}_{-}^{1}$ is the stable small resolution.

details of the global embedding. In this case, the phenomena discussed above lead to dynamical topology change of the geometry, with the intersection numbers of cycles changing, as for orbifold flips [8, 9]. Our analysis of the physics here has been somewhat indirect, using linear sigma models: in particular it would be useful to construct the localized tachyons explicitly 444. From the above analysis, we make the following statements and speculations (see Figure 3 for a heuristic picture), with intuition based on spacetime perspectives of the physics rather than e.g. worldsheet RG flows.

Given the obstructions to 3-cycle deformations, the phases of flip conifolds simply consist of the small resolutions, bundles over $\mathbb{P}_{ \pm}^{1}$ with possible residual $\mathbb{C}^{3} / G$ singularities. Such a singularity decays to its stable small resolution: since the dynamics forces the 2-cycles to expand in time (as is seen from the flow to large Kähler parameter $r$ in the GLSM of the previous section), this means that these unstable conifolds basically decay to smooth spaces, since the possible residual orbifold singularities if unstable will themselves decay to smooth spaces too [8]. There is an inherent directionality in time in the dynamics here. Say at early times one sets up the system in the unstable small resolution (which is a semiclassical phase if the 2-cycle volume is large, i.e. large $r$ ): then the dynamics dictates a flip transition towards the stable one executing mild topology change ${ }^{12}$ in the process. There appears to be no analog here of the phenomena associated with strong topology change [27] 28]: the small resolutions simply replace one 2-sphere with another so that while the intersection numbers of various cycles in a compact embedding in general change under the corresponding flip transition, the Euler number does not. We have been largely using classical geometry to study deformations so far:

\footnotetext{
${ }^{12}$ We are using "mild" and "strong" along the lines of e.g. [29].
} 
one could therefore ask if conifold transitions could occur nonperturbatively since spacetime supersymmetry is broken. In other words, we ask whether new 3-cycle branches open up nonperturbatively close to the transition point causing drastic tears in spacetime. While we cannot rule it out, this seems unlikely since $(2,2)$ worldsheet supersymmetry is unbroken and worldsheet instantons ensure that there is a $|\log \sigma|^{2}$ growth (42) in the potential energy given by the quantum twisted chiral superpotential. Thus unlike the supersymmetric conifold, there are no singularities in the Higgs branch where new 3-cycle (continuous Coulomb) branches can open up along the RG flow direction (which also suggests that the abstract deformations described at the end of Sec. 3.2.1 are physically unlikely): in other words, the quantum 2-cycle volumes are not vanishing and one does not expect sufficiently light wrapped brane states. It is worthwhile noting that the dynamics of a flip is quite different from a time-varying flop mediated by say a slowly varying modulus: by the $(R G)$ time the evolution of the geometry crosses over between phases, the situation is far from a slow variation and is rather a rapid transition between phases of distinct topology. Thus the rate at which the geometry is evolving in time (in spacetime) "near" the transition itself is large, and the region near the singularity where quantum corrections are large is a transient intermediate state. It would be interesting to investigate in detail the dynamics here in the context of a compact embedding with fluxes, in part to understand if there are analogs here of "moduli" trapping [45] and other phenomena.

Consider now the dynamics of the compact space in which unstable singularities are embedded: a nonsupersymmetric orbifold of $\mathbb{C P}^{3}$ would typically contain isolated $\mathbb{C}^{3} / \mathbb{Z}_{N}$ singularities. From a linear sigma model analysis, we see that $\mathbb{C P}^{3}$ has a tendency to spontaneously shrink its overall volume (a nice discussion of this can be found in e.g. [30]). Then one imagines that the evolution ${ }^{13}$ of an orbifold of $\mathbb{C P}^{3}$ would depend on the competition between this tendency to spontaneously shrink and the effects of possible tachyonic cycles expanding as a result of condensation of closed string tachyons localized to the isolated $\mathbb{C}^{3} / \mathbb{Z}_{N}$ singularities: a naive expectation is that the overall tendency to collapse perhaps dominates the expanding tachyons essentially since the collapse is a bulk effect whereas the tachyons are localized objects associated to cycles of some nonzero codimension, resulting in a collapsed $\mathbb{C P}^{3}$ as the late time endpoint ${ }^{14}$. However now imagine turning on some fluxes: then the effective mass for a twisted state tachyon $T$ increases as $m_{e f f}^{2}=-m_{0}^{2}+|F|^{2}$, from the coupling of the fluxes to the twisted

\footnotetext{
${ }^{13}$ Note that $\mathbb{C P}^{3}$, with nonzero curvature, is not a solution by itself to the string equations of motion: what we imagine here is that an appropriate solution can be constructed in terms of a nontrivial FRW-like cosmology in the remaining $4 \mathrm{D}$ spacetime, where the evolution of the $4 \mathrm{D}$ scale factor reflects that of the internal space, somewhat along the lines of the cosmological solutions in e.g. [46].

${ }^{14}$ This argument is perhaps too glib when the overall size of the $\mathbb{C P}^{3}$ shrinks to substringy length scales, where a more careful analysis going beyond geometry is required.
} 
sector states [47. Thus maybe more interesting and useful endpoints could result if appropriate fluxes stabilize both localized tachyon masses and the overall dynamical volume. A Calabi-Yau orbifold embedding of a nonsupersymmetric $\mathbb{C}^{3} / \mathbb{Z}_{N}$ singularity has no obvious such tendency to spontaneously shrink so one might naively expect that it simply evolves spontaneously towards a large volume limit with the expanding tachyonic cycles. On the other hand, one might expect that an unstable flip conifold can be embedded in an appropriate nonsupersymmetric orbifold of a Calabi-Yau that develops a supersymmetric conifold singularity, with the orbifold action on the local supersymmetric conifold singularity resulting locally in the flip conifold singularity in question. However there may be constraints on the $n_{i}$ defining the singularity for the existence of such an embedding in a space that is locally Calabi-Yau (but with global identifications): i.e. not all such local singularities may admit locally supersymmetric string compactifications. It would be worth exploring the dynamics of these systems further.

Acknowledgments: It is a pleasure to thank D. Morrison for collaboration in the incipient stages of this work as well as several useful discussions. I have also benefitted from useful discussions with S. Ashok, C. Beasley, A. Dabholkar, R. Gopakumar, S. Govindarajan, T. Jayaraman, S. Mukhi, N. Nitsure, A. Sen and C. Vafa. I'd like to thank the Organizers of the "Mathematical Structures in String Theory" Workshop and the hospitality of the KITP Santa Barbara, USA, and the Harvard Theory Group, USA, as this paper was being finalized. This research was supported in part by the National Science Foundation under Grant No. PHY99-07949.

\section{A More on deformations}

Consider a nonminimal flip region, i.e. at most one of the $n_{i}$ is equal to one: say $n_{1}=1$. We now look for cases when $\nu=1+\frac{1}{n_{2}}+\frac{1}{n_{3}}+\frac{1}{n_{4}} \in \mathbb{Z}$, i.e. the phase is trivial. Since $\nu>1$, we need to look for $n_{i}$ such that $\nu=2,3$. We see that any two integers $n_{3}, n_{4}$ satisfying $n_{2}=\frac{n_{3} n_{4}}{(\nu-1) n_{3} n_{4}-n_{3}-n_{4}}$ such that $n_{2} \in \mathbb{Z}^{+}$give $\nu=2,3$ and therefore give such nonminimal flip regions. These have charge matrices (with $\nu=2,3$ a parameter)

$$
Q=\left(\begin{array}{llll}
1 & \frac{n_{3} n_{4}}{(\nu-1) n_{3} n_{4}-n_{3}-n_{4}} & -n_{3} & -n_{4}
\end{array}\right) \equiv\left(\begin{array}{cccc}
1 & K & -n_{3} & -n_{4}
\end{array}\right), \quad n_{3}, n_{4} \in \mathbb{Z}^{+} .
$$

For this singularity to be isolated, we must have $n_{3}, n_{4}, K$ to be positive integers coprime w.r.t. each other. Let $n_{3}, n_{4}$ to be written via their prime factors as $n_{3}=p_{1} \cdot p_{2} \ldots p_{k}, n_{4}=q_{1} \cdot q_{2} \ldots q_{l}$. 
For $n_{3}, n_{4}$ to be coprime, all the $p_{i}, q_{j}$ must be coprime. Then we want

$$
K=\frac{n_{3} n_{4}}{(\nu-1) n_{3} n_{4}-n_{3}-n_{4}}=\frac{\left(p_{1} p_{2} \ldots p_{k}\right)\left(q_{1} q_{2} \ldots q_{l}\right)}{\left((\nu-1) p_{1} \ldots p_{k} \cdot q_{1} \ldots q_{l}\right)-\left(p_{1} \ldots p_{k}\right)-\left(q_{1} \ldots q_{l}\right)}
$$

to be a positive integer and coprime to the two factors in the numerator. If the denominator divides the numerator, then it must be that some of the numerator $p_{i}, q_{j}$ factors cancel (using prime factorization of the denominator). Then we have $K=\prod\left(p_{i}^{\prime} q_{j}^{\prime}\right)$, the residual product (deleting the cancelled primes). Thus $K$ has common factors with $n_{3}, n_{4}$, i.e. the residual primes $p_{i}^{\prime}, q_{j}^{\prime}$ - hence non-isolated. More generally consider none of the $n_{i}$ to be equal to one. Then for say $\nu=1$, using (22) we have $n_{4}=\frac{n_{1} n_{2} n_{3}}{n_{1} n_{2} n_{3}-n_{2} n_{3}-n_{3} n_{1}-n_{1} n_{2}}$, so that if $n_{4} \in \mathbb{Z}$, then as above it is not coprime with $n_{1}, n_{2}, n_{3}$, hence not isolated. Similarly, if $\nu=2,3$, we have $n_{4}=\left(n_{1} n_{2} n_{3}\right) /(\ldots)$, hence not isolated. Similarly consider the case when at least two of the $n_{i}$ are equal to one, i.e. a minimal flip region ${ }^{15}$, say $n_{1}, n_{2}=1$, with the charge matrix

$$
Q=\left(\begin{array}{llll}
1 & 1 & -n_{3} & -n_{4}
\end{array}\right)
$$

For the phase to be trivial, we want $\nu=2+\frac{1}{n_{3}}+\frac{1}{n_{4}} \in \mathbb{Z}$. The pair $\left(n_{3}, n_{4}\right)=(2,2)$ giving $\nu=1$ is the charge matrix $Q=\left(\begin{array}{llll}1 & 1 & -2 & -2\end{array}\right)$, with $\sum Q_{i}=-2 \neq 0$. More generally, we can see that this is the unique such region in this case: since $\nu>2$ here, $\nu=3$ implies $\frac{1}{n_{3}}+\frac{1}{n_{4}}=1$, i.e. $n_{4}=\frac{n_{3}}{n_{3}-1}$, which is integral only if $\left(n_{3}-1\right)$ divides $n_{3}$, i.e. $n_{3}=2, n_{4}=2$. This shows that there are no deformations whenever the $n_{i}$ are not of this form.

\footnotetext{
${ }^{15}$ For instance, in the singularity of Sec. 2 and Sec. 3.1 , we had $Q=\left(\begin{array}{cccc}1 & 2 & -1 & -3\end{array}\right)$.
} 


\section{References}

[1] A. Adams, J. Polchinski, E. Silverstein, "Don’t panic! Closed string tachyons in ALE spacetimes", hep-th/0108075.

[2] C. Vafa, "Mirror symmetry and closed string tachyon condensation", hep-th/0111051.

[3] J. Harvey, D. Kutasov, E. Martinec, G. Moore, "Localized tachyons and RG flows" hep-th/0111154.

[4] E. Martinec, "Defects, decay and dissipated states", hep-th/0210231.

[5] M. Headrick, S. Minwalla, T. Takayanagi, "Closed string tachyon condensation: an overview", hep-th/0405064.

[6] E. Martinec, G. Moore, "On decay of K-theory", hep-th/0212059.

[7] G. Moore and A. Parnachev, "Localized tachyons and the quantum McKay correspondence", hep-th/0403016.

[8] David R. Morrison, K. Narayan, M. Ronen Plesser, "Localized tachyons in $\mathbb{C}^{3} / \mathbb{Z}_{N}$ ", hep-th/0406039.

[9] David R. Morrison, K. Narayan, "On tachyons, gauged linear sigma models and flip transitions", hep-th/0412337.

[10] T. Sarkar, "On localized tachyon condensation in $\mathbb{C}^{2} / \mathbb{Z}_{N}$ and $\mathbb{C}^{3} / \mathbb{Z}_{N}$ ", hep-th/0407070.

[11] P. Aspinwall, B. Greene, D. R. Morrison, "Calabi-Yau moduli space, Mirror manifolds and spacetime topology change in string theory", hep-th/9309097.

[12] E. Witten, "Phases of $\mathcal{N}=2$ theories in two dimensions", hep-th/9301042.

[13] I. Melnikov, M. Ronen Plesser, "The Coulomb branch in gauged linear sigma models", hep-th/0501238.

[14] A. Adams, X. Liu, J. McGreevy, A. Saltman, E. Silverstein, "Things fall apart: topology change from winding tachyons", hep-th/0502021.

[15] G. Moore and A. Parnachev, "Profiling the brane drain in a nonsupersymmetric orbifold", hep-th/0507190.

[16] I. Melnikov, M. Ronen Plesser, "A-model correlators from the Coulomb branch", hep-th/0507187.

[17] J. McGreevy, E. Silverstein, "The tachyon at the end of the universe", hep-th/0506130. 
[18] H. Yang, B. Zwiebach, "Rolling closed string tachyons and the big crunch", hep-th/0506076.

[19] H. Yang, B. Zwiebach, "A closed string tachyon vacuum ?", hep-th/0506077.

[20] G. Horowitz, "Tachyon condensation and black strings", hep-th/0506166.

[21] S. Ross, "Winding tachyons in asymptotically supersymmetric black strings", hep-th/0509066.

[22] A. Dymarsky, I. Klebanov, R. Roiban, "Perturbative gauge theory and closed string tachyons", hep-th/0509132.

[23] D. Kutasov, "Accelerating branes and the string/black hole transition", hep-th/0509170.

[24] E. Silverstein, "Dimensional mutation and spacelike singularities", hep-th/0510044.

[25] O. Bergman, S. Hirano, "Semi-localized instability of the Kaluza-Klein linear dilaton vacuum", hep-th/0510076.

[26] P. Candelas, X. de la Ossa, "Comments on conifolds", Nucl. Phys. B342, 246 (1990).

[27] A. Strominger, "Massless black holes and conifolds in string theory", hep-th/9504090.

[28] B. Greene, D. R. Morrison, A. Strominger, "Black hole condensation and the unification of string vacua", hep-th/9504145.

[29] B. R. Greene, "String theory on Calabi-Yau manifolds", hep-th/9702155.

[30] E. Witten, in Quantum fields and strings: a course for mathematicians, see, e.g., Lecture 12 at http://www.cgtp.duke.edu/QFT/spring/.

[31] D. R. Morrison, M. R. Plesser, "Summing the instantons: quantum cohomology and mirror symmetry in toric varieties", hep-th/9412236.

[32] David R. Morrison, M. Ronen Plesser, "Nonspherical horizons", hep-th/9810201.

[33] T. Hubsch, "Calabi-Yau Manifolds: A Bestiary for Physicists", World Scientific Publishing Co., 1992.

[34] D. Ghoshal, D. Jatkar, S. Mukhi, "Kleinian singularities and the ground ring of $c=1$ string theory", hep-th/9206080.

[35] K. Dasgupta, S. Mukhi, "Brane constructions, conifolds and M theory", hep-th/9811139.

[36] A. Uranga, "Brane configurations for branes at conifolds", hep-th/9811004.

[37] M. Schlessinger, "Rigidity of quotient singularities," Invent. Math. 14, 17 (1971). 
[38] B. Ezhuthachan, S. Govindarajan, T. Jayaraman, "A quantum McKay correspondence for fractional 2p-branes on LG orbifolds", hep-th/0504164.

[39] R. Gopakumar, C. Vafa, "Branes and fundamental groups", hep-th/9712048.

[40] K. Altmann, "Toric $\mathbb{Q}$-Gorenstein singularities", alg-geom/9403003, "The versal deformation of an isolated toric Gorenstein singularity", alg-geom/9403004, "One-parameter families containing 3-dimensional toric Gorenstein singularities", alg-geom/9609006.

[41] D. Ghoshal, P. Lakdawala, S. Mukhi, "Perturbations of the ground varieties of $c=1$ string theory", hep-th/9308062.

[42] I. Klebanov, E. Witten, "Superconformal field theory on threebranes at a Calabi-Yau singularity", hep-th/9807080.

[43] M. Douglas, G. Moore, "D-branes, quivers and ALE instantons", hep-th/9603167.

[44] Work in progress.

[45] L. Kofman, A. Linde, X. Liu, A. Maloney, L. McAllister, E. Silverstein, "Beauty is attractive: moduli trapping at enhanced symmetry points", hep-th/0403001.

[46] P. Chen, K. Dasgupta, K. Narayan, M. Shmakova, M. Zagermann, "Brane inflation, solitons and cosmological solutions: I" hep-th/0501185.

[47] E. Silverstein, "Counter-intuition and scalar masses," hep-th/0407202. 